\title{
Quality of life, depression and fatigue in patients with multiple
} sclerosis

\author{
Eleni Lazaridou*1, Konstantina Mantziava ${ }^{1}$, Ria Pita ${ }^{1}$, Grigoris Kiosseoglou ${ }^{1}$, \\ Adamantia Dourouma ${ }^{1}$ and Anastassios Orologas ${ }^{2}$
}

Address: ${ }^{1}$ School of Psychology, Aristotle University of Thessaloniki, Thessaloniki, Greece and ${ }^{2}$ School of Medicine, 2nd Department of Neurology, Aristotle University of Thessaloniki, Thessaloniki, Greece

* Corresponding author

from International Society on Brain and Behaviour: 2nd International Congress on Brain and Behaviour

Thessaloniki, Greece. 17-20 November 2005

Published: 28 February 2006

Annals of General Psychiatry 2006, 5(Suppl I):SI56 doi:I0.II86/I744-859X-5-SI-SI 56

\section{Background}

The present study aimed at the longitudinal evaluation of the quality of life (QoL) of Greek patients - suffering from definite clinical multiple sclerosis (MS) - and its relation with fatigue and depression, in a time span of two years.

\section{Materials and methods}

In the first examination (2003) seventy seven participants (42 women and 35 men) with MS, aged 17-67 years, were administered: a) QOLIE-89, measuring QoL and functioning levels, b) BDI measuring depression and c) FIP and FSS scales, measuring 4 dimensions of "fatigue" and "severity of fatigue", respectively. Two years later (2005) fifty of those patients were randomly selected and were administered the above-mentioned tests for a second time. Twenty six patients, who were receiving antidepressants during either the first or the second evaluation, were excluded from the reevaluation study. Eventually, the final group consisted of twenty four patients (14 women and 10 men), aged $20-72$ years.

\section{Results}

In the first evaluation (2003), the Pearson's correlation showed that the 4 dimensions and "severity of fatigue" correlated (negatively) with most subscales of QOLIE-89. Stepwise regression analysis showed that physical and cognitive/mental fatigue predicted different aspects of MS patients" QoL. Depression predicted health-related aspects of QoL and in some cases added to the effects of fatigue on QoL.

In the second evaluation (2005), according to the analyses through ANOVA models, a statistically important improvement in the general quality of life was revealed, but no other statistically important changes concerning the measurement of depression (BDI) and the dimensions of "fatigue" and "severity of fatigue" (FIP, FSS) were found.

\section{Discussion}

The results of our study on the negative influence of depression and fatigue on the QoL of patients with MS are in accordance with existing literature. Regarding the outcomes from the follow-up examination it is noteworthy that there were no statistically important changes in the measurement of depression or fatigue, but there was an important amelioration in the general quality of life. This could be due to the two-year interval between the two examinations or due to the acceptance, better confrontation and coping with the disease and its course by the patients.

\section{References}

I. Egner A, Phillips VL, Vora R, Wiggers E: Depression, fatigue, and health- related quality of life among people with advanced multiple sclerosis: Results from an exploratory rehabilitation study. Neurorehabilitation 2003, 18:125-133.

2. Janardhen V, Bakshi R: Quality of life in patients with multiple sclerosis: The impact of fatigue and depression. Journal of the Neurological Sciences 2002, 205:5I-58.

3. Daly E, Komaroff AL, Bloomingdale K, et al.: Neuropsychological Function in Patients with Chronic Fatigue Syndrome, Multiple Sclerosis, and Depression. Applied Neuropsychology 200I, 8:12-22.

4. Marrie A, Fisher A, Miller D, Lee J, Rudick R: Association of fatigue and brain atrophy in multiple sclerosis. Journal of the Neurological Sciences 2005, 228:161-166. 